\title{
MYTHEN detector - perspectives in residual stress measurements
}

\author{
Dubravka Šišak Jung ${ }^{1, a^{*}}$, Lasse Suominen ${ }^{2, b}$, Jari Parantainen ${ }^{2, c}$, \\ Christoph Hörmann ${ }^{1, d}$ \\ ${ }^{1}$ DECTRIS Ltd, Neuenhoferstrasse 107, 5400 Baden, Switzerland \\ ${ }^{2}$ Stresstech Oy, Tikkutehtaantie 1, 40800 Vaajakoski, Finland \\ adubravka.sisak@dectris.com, bLasse.Suominen@stresstech.com, \\ cJari.Parantainen@stresstech.com, dchristoph.hoermann@dectris.com
}

Keywords: microstrip detector, MYTHEN, stress measurement optimization, fluorescence

\begin{abstract}
MYTHEN is a single-photon-counting strip detector. Its main features are high spatial resolution, zero noise, fluorescence suppression, fast readout, high dynamic range, radiation-hard and maintenance-free design. Perspectives of such a detector in residual stress measurements involve: (i) Measurements of absorbing/thick materials (ii) Well resolved peaks (iii) excellent signal-to-noise ratio (iv) Analysis of alloys (v) Fast data collection (vi) Accurate low content retained austenite measurements (vii) in situ measurements and mapping (viii) infinite life cycle. Technical details and application in synchrotron and laboratory diffractometers will be presented.
\end{abstract}

\section{Introduction}

First attempts to solve industrial processes by stress measurements using X-ray diffraction (XRD) technique were carried out already in the 1950-ies [1,2]. Since then, many approaches have been devised, and nowadays the method is successfully applied in almost every branch of industry. Such a broad implementation was possible mostly owing to the development of XRD diffractometers. Both laboratory and portable machines are commonly used with $\mathrm{Cr}$-, $\mathrm{Mn}$ - or $\mathrm{Cu}$ - sources and a variety of X-ray detectors. Portable equipment has strongly expanded industrial stress measurement by circumventing the limitation of sample size. However, limitations of X-ray detectors often cause industry requirements not to be fulfilled. First, better spatial resolution and smaller phase contents (retained austenite content under 1\%) are warranted. Second, Fitzpatrick et al [3] prescribe high intensity peaks (1000 counts) with 20 points per peak and high signal-to-noise ratio to be used for the analysis. In order to obtain such counting statistics, measurements should either be repeated, or the exposure time should be longer. In contrast to that, industrial application requires measurements to be as short as possible. Compromise between these requirements and the data quality depends mostly on detector that is used. Type of the detector is especially important in analysis of materials with strong fluorescence or small phase content. In this work, we explore the application of state-ofthe-art detectors for residual stress measurements. These detectors are based on hybrid pixel/ microstrip technology and their basic construction involves silicon sensors and CMOS (complementary metal-oxide semiconductor) readout ASICs (application specific integrated circuit). This results in a new, single-photon-counting mode and extremely fast readout. That is, due to the absence of detector noise, excellent signal-to-noise ratio can be obtained in impressively reduced experimental time. This opens up new possibilities in residual stress and retained austenite analyses. Limitations imposed by the sample (fluorescence, coated samples, texture, alloys, large grains, small phase content etc.) can be overcome. Also, new methods such as in situ measurements for following industrial processes and sample mapping are feasible.

\section{Detectors - an overview}

Residual stress measurements traditionally rely on different types of detectors. Depending on the data-collection mode, these can be grouped in point, position sensitive and area detectors. 
Point detectors. This group contains proportional and scintillation detectors. The chosen $2 \theta$ range is scanned. Thus in order to obtain satisfying statistics, measurement times can become dramatically long. Moreover, scintillation detectors are not very efficient at long wavelengths (Cr-radiation) [4]. Position sensitive detectors. Portable diffractometers have three type of line detectors: position sensitive proportional counters, scintillation detectors with photomultiplier tube and solid state (NMOS) spectrometer detectors. In general, line detectors collect data over a certain $2 \theta$ range (usually $15^{\circ}$ ) simultaneously, what can significantly reduce the data acquisition time. Among these, strong point of NMOS detector is simplicity and commercial availability of driving electronic and detector itself. Still, it is designed for spectrometer applications in visible light range, resulting in lower efficiency for X-ray radiation when compared to specially designed new detectors.

Area detectors. This type of detectors provides 2D data (Debye rings). Their most obvious advantage is that additional information about the possible texture or grain size can be obtained. On the other hand, they are reported to be bulky, fragile and easily saturated with sample fluorescence.

Single-photon-counting detectors. Single-photon-counting detectors are pixel or microstrip arrays of silicon sensors, with CMOS readout ASICs. If the measurement is depicted as a deposition of Xrays randomly in time, such a detector measures each photon independently. The photon coming to Si-sensor is directly converted into electric charge, so no dark current is present. In order to suppress fluorescence and noise, user-defined energy threshold $\left(E_{\mathrm{thr}}\right)$ is set. The optimal choice of $E_{\mathrm{thr}}$ is about $1 / 2$ of the value of energy of the radiation used. Each pixel or strip in this array can count up to $10^{6}$ photons per second, and the signal readout is in milisecond range. These detectors were initially made for high energy physics. This radiation-hard design now allows virtually infinite life cycle. One of the first detectors of that kind adapted for X-ray measurements was 2D pixel detector PILATUS, developed at Paul Scherrer Institute (PSI) for protein crystallography [5, 6]. The 1D strip detector MYTHEN was developed for the time-resolved X-ray powder diffraction (XPD) measurements [7]. Both detectors are commercialized through DECTRIS Ltd, Baden, Switzerland.

MYTHEN. The basic structure of MYTHEN contains 1280 silicon strips, arranged in an 1D array. Such an assembly is referred to as module. Each of these strips is $50 \mu \mathrm{m}$ wide and acts as a detector, counting up to $10^{6}$ photons per second. The corresponding point-spread function is one channel (strip), resulting in peaks with minimal widths. Two further important features are extremely high dynamic range (24 bits), and a readout time of $0.3 \mathrm{~ms}$. While the first allows both high and low intensity peaks $\left(1: 10^{7}\right)$ to be measured in the same scan, the latter enables short acquisition times. Modules have flexible design, so that various needs can be fulfilled. Namely, sensor thickness can be chosen so that it shows highest efficiency for a chosen energy range. Moreover, several modules can be assembled in 1D array, resulting in multimodular systems that can cover significantly wider angle range (up to $120^{\circ}$ in $2 \theta$ ).

MYTHEN applications. Shortly after the first instrument (24 modules, 24K system) was installed at Swiss Light Source (SLS) [8], time-resolved strain measurements were performed. In particular, deformations in coarse-grained nanocrystalline nickel were followed in situ, and subsequently residual dislocations described [9]. This was followed by series of in situ stress measurements carried out at Karlsruhe Institute of Technology, where two monomodular (1K) MYTHEN detectors were employed in $\sin ^{2} \psi$ method [10-11]. While the implementation of both MYTHEN $1 \mathrm{~K}$ and 24K systems in stress measurements on synchrotron sources yielded excellent results, laboratory measurements are still not described. In the following paragraphs, measurements carried out in collaboration between DECTRIS Ltd. and Stresstech Oy are reported.

\section{Experimental.}

All measurements were performed on standard Stresstech diffractometer. In order to evaluate the detector efficiency and optimize the experiment, NMOS type array detector and DECTRIS' MYTHEN 1K detector were used (Table 1). Both detectors were installed on the goniometer simultaneously, with about the same distance to the investigated material, to ensure similar 
measurement conditions. Several measurements were carried out: (i) evaluation of exposure time necessary to obtain a quality XRD peak (ii) repeat tests for calculating the deviation (accuracy) of peak position determination (iii) residual stress measurement.

Table 1 Technical specifications of MYTHEN 1K and NMOS detector

\begin{tabular}{|l|l|l|}
\hline Specification & MYTHEN 1K & NMOS \\
\hline Detection & Single-photon counting & integrating \\
\hline No. strips/pixels & $1280(1$ module) & 512 \\
\hline Strip/pixel width $[\mu \mathrm{m}]$ & 50 & 50 \\
\hline Strip/pixel length $[\mathrm{mm}]$ & 8 & 2.5 \\
\hline Sensor thickness $[\mu \mathrm{m}]$ & 320 & 4 \\
\hline Efficiency for Cu-radiation $[\%]$ & 97.1 & 5.7 \\
\hline Efficiency for Cr-radiation $[\%]$ & 93.9 & 17 \\
\hline $\begin{array}{l}\text { Maximum count rate per strip } \\
\text { [cts/s] }\end{array}$ & $10^{6}$ & n/a \\
\hline
\end{tabular}

\section{Exposure time tests}

The aim of these tests was to examine the exposure time necessary to detect diffraction peaks, whose quality is sufficient for peak-fitting procedure. Three different samples, titanium, aluminium powder and lead sheet were measured on the standard Stresstech diffractometer, using Cu-radiation and Ni filter. These measurements were then repeated with MYTHEN 1K detector, without filter.

\section{Titanium}

Titanium is important aerospace material. Traditionally it is measured by $\mathrm{Cu}$ radiation which creates exceptional strong fluorescence. In NMOS experiment, the sample was illuminated for $4 \mathrm{~s}$, and characteristic peak at 360 pixel $\left(2 \theta=148^{\circ}\right)$ was not observed. Once the exposure was increased 5 times, the peak became detectable. Due to the fluorescence suppression, MYTHEN data resulted in prominent peak (450 pixel) and good signal-to-noise ratio after exposure of only $2 \mathrm{~s}$ (Figure 1a,b). These results suggest that fluorescence suppression (energy threshold) in MYTHEN could be utilized in fast measurements of absorbing materials, alloys and coated samples.

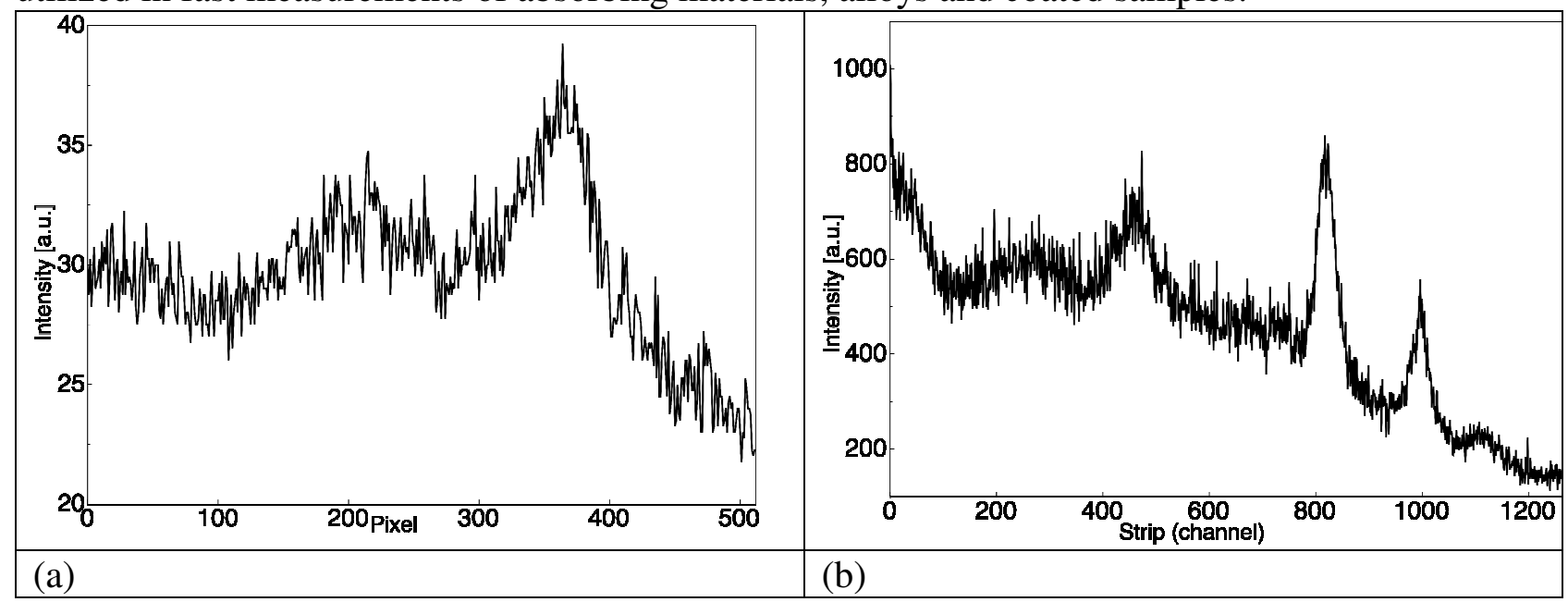

Figure 1 Measurement of titanium sample using the $\mathrm{Cu}$-radiation and (a) NMOS detector, Ni-filter and exposure time of $20 \mathrm{~s}$ (b) MYTHEN 1K detector, no filter and exposure time of $2 \mathrm{~s}$.

Al powder

Comparison of the two datasets, obtained by measurement of aluminium sample with NMOS detector $(t=40 \mathrm{~s})$ and MYTHEN $1 \mathrm{~K}(t=1 \mathrm{~s})$ reveal that excellent signal-to-noise ratio can be achieved with MYTHEN detector, although the exposure time is 40 times less (Figure 2a, b). As previously shown in synchrotron application, significant reduction of data acquisition coupled with no-noise data could be implemented in laboratory for fast in situ measurements. 


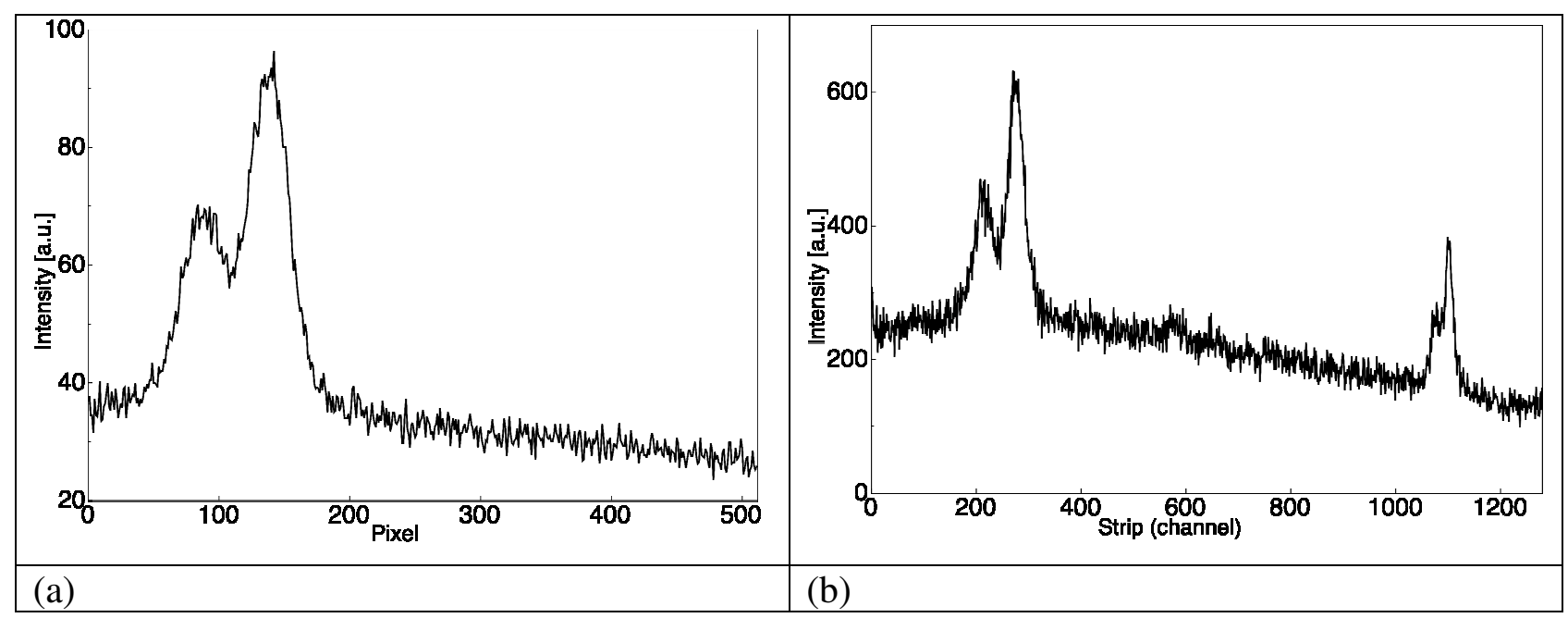

Figure 2 Comparison of the data collected using the $\mathrm{Cu}$-radiation with (a) NMOS detector, Ni-filter, $40 \mathrm{~s}$ exposure time and (b) MYTHEN $1 \mathrm{~K}$ detector, no filter, $1 \mathrm{~s}$ exposure time.

$\mathrm{Pb}$ sheet

Similar to the previous case, comparison of measurement of $\mathrm{Pb}$ sheet with NMOS detector $(t=40 \mathrm{~s})$ and MYTHEN 1K $(t=5 \mathrm{~s})$ reveals prominent peak and excellent signal-to-noise ratio in MYTHEN data, although the exposure time is 8 times less (Figure $3 a, b)$.

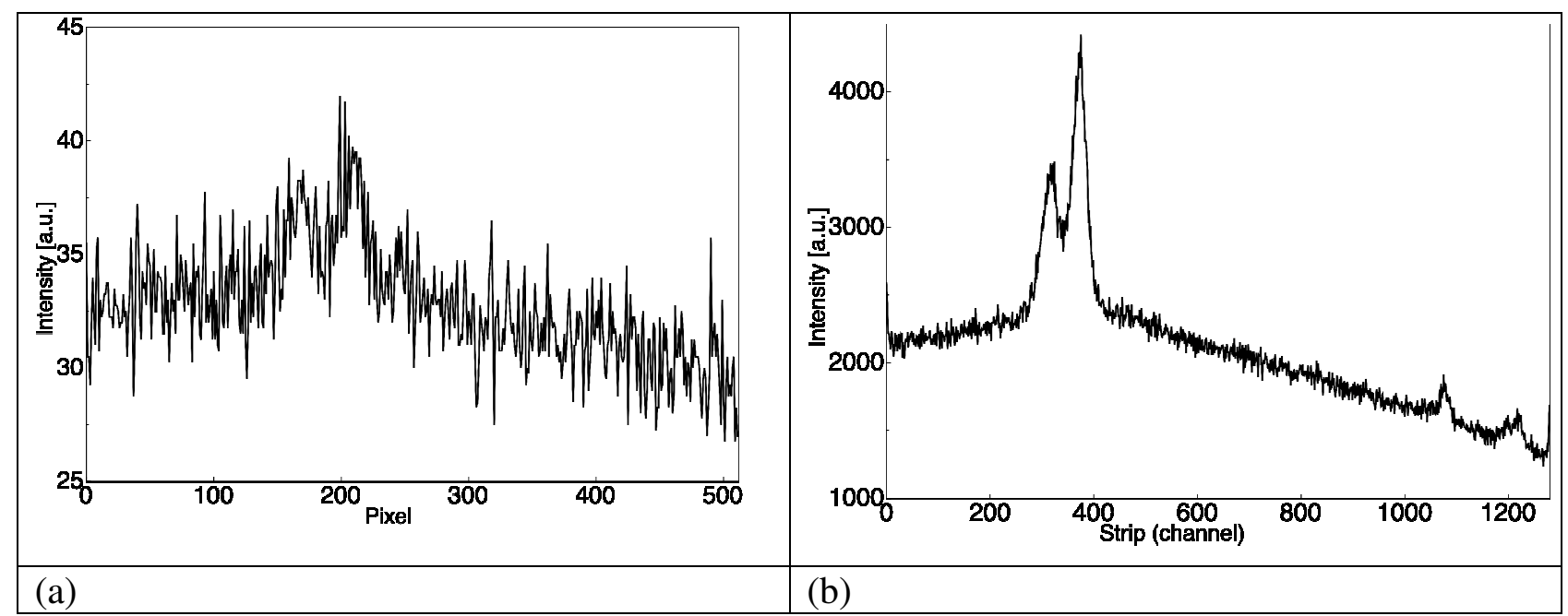

Figure 3 Data collected using Cu-radiation with (a) NMOS detector, Ni-filter, 40 s exposure time and (b) MYTHEN $1 \mathrm{~K}$ detector, no filter, $5 \mathrm{~s}$ exposure time.

\section{Standard deviation (accuracy) in determination of peak position}

The accuracy of the peak position determination depends on many factors. These can be approximated as a requirement that the peak maximum should be prominent (number of counts, number of measured points). Here, a good signal-to-noise ratio is of extreme importance. Signal-tonoise ratio, on the other hand, depends on fluorescence, noise level and exposure time. In this experiment, a ferrite powder sample was measured 10 times using Cr-radiation with NMOS and MYTHEN 1K detector, each time with a different exposure time. Peak position and its deviation $(1 \sigma)$ as pixel $\left(1\right.$ pixel $\left.=0.03^{\circ}\right)$ was calculated as a function of the exposure time. Figure 4 shows that for the same level of accuracy, MYTHEN detector requires 10 times less exposure time.

In order to examine the accuracy of the measurements of fluorescent samples, the standard deviation of the peak position determination was also calculated for the data collected on titanium sample, using $\mathrm{Cu}$-radiation (Figure 5). 


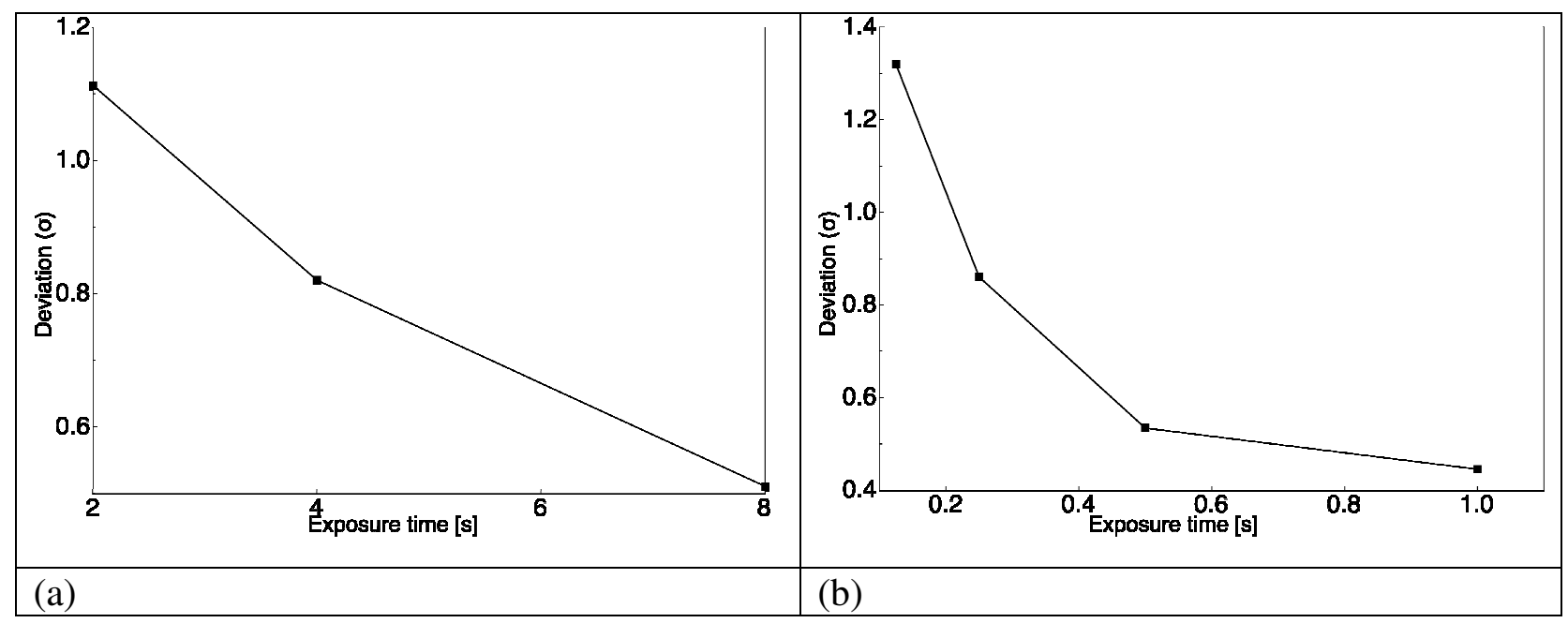

Figure 4 Standard deviation of peak position determination as a function of exposure time. Ferrite powder measured using Cr-radiation and (a) NMOS detector and (b) MYTHEN 1K detector.

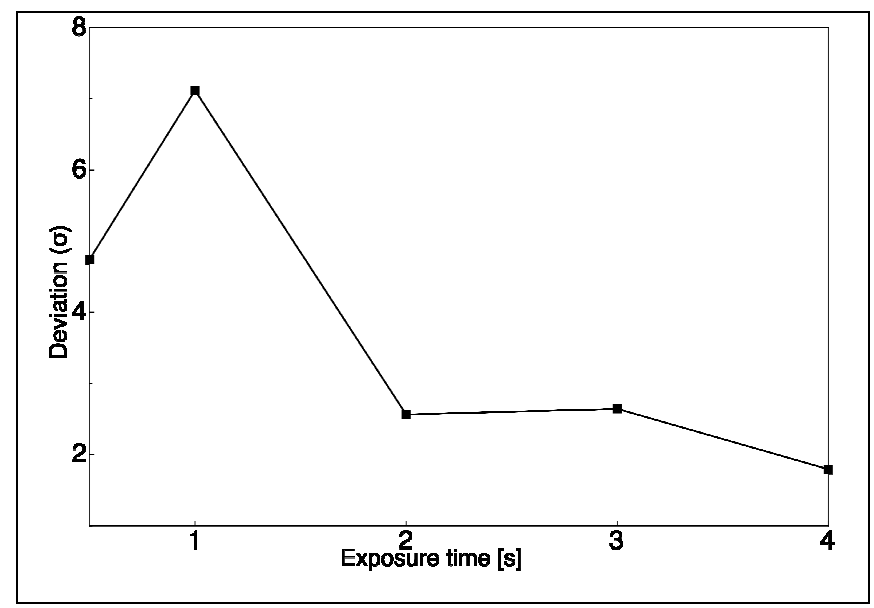

Figure 5 Standard deviation of peak position determination as a function of exposure time. Titanium sample measured using $\mathrm{Cu}$-radiation and MYTHEN 1K detector.

\section{Residual stress measurements}

A titanium sample was used for residual stress measurements with MYTHEN $(t=2 \mathrm{~s})$ and NMOS $(t$ $=20 \mathrm{~s}$ ) detectors using $\mathrm{Cu}$-radiation. The collected data was processed using Origin7, and the obtained results were found to be comparable (Figure 6a, b).

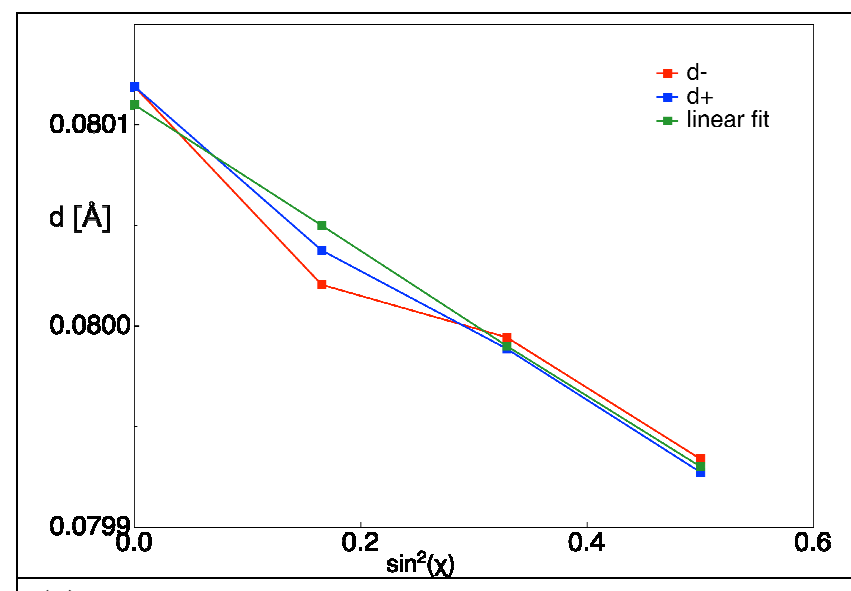

(a)

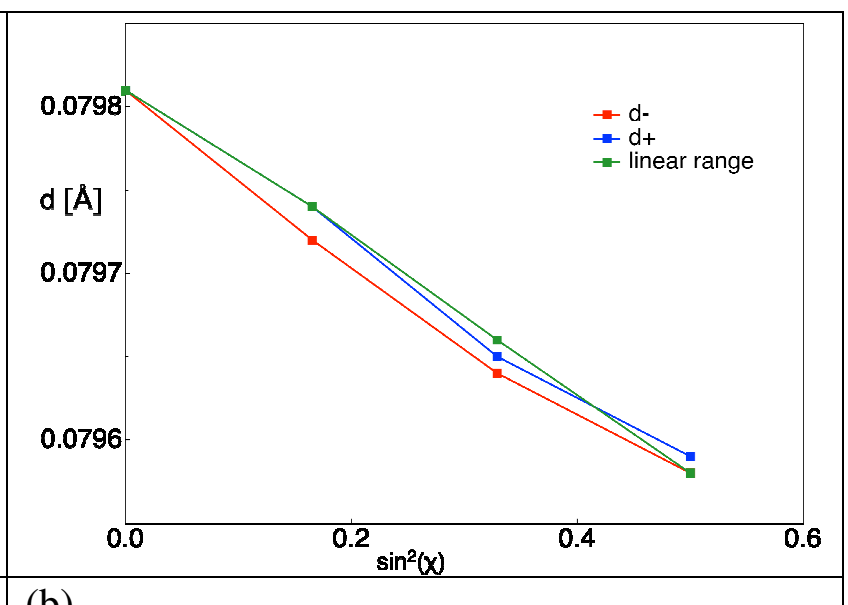

(b)

Figure 6 Results of residual stress measurement of titanium sample (reflection 302) using Curadiation and (a) NMOS detector (b) MYTHEN 1K detector 


\section{Summary}

Comparison of the several measurements performed on three samples ( $\mathrm{Al}, \mathrm{Pb}, \mathrm{Ti}$ ) showed some advantages of MYTHEN detector. In particular, the measurement time can be reduced up to 40 times, without compromising the data statistics. Moreover, in order to obtain similar accuracy of peak position determination, MYTHEN requires 10 times less exposure time than the standard NMOS detector. Especially in the case of strong fluorescence as Ti-samples with Cu-radiation, MYTHEN detector is superior: faster and more accurate residual stress measurements can be performed. Additionally, MYTHEN shows no wear due to X-ray exposure.

Table 2 Advantages of MYTHEN detector

\begin{tabular}{|l|l|l|l|l|l|}
\hline Sample & K-edge $[\mathrm{keV}]$ & $\mathrm{E}[\mathrm{keV}]$ & NMOS $t[\mathrm{~s}]$ & MYTHEN $t[\mathrm{~s}]$ & MYTHEN $E_{\text {thr }}[\mathrm{keV}]$ \\
\hline $\mathrm{Ti}$ & 4.51 & $\mathrm{Cu}(8.048)$ & 20 & 2 & 6.4 \\
\hline $\mathrm{Al}$ & 1.49 & $\mathrm{Cu}(8.048)$ & 40 & 1 & 6.4 \\
\hline $\mathrm{Pb}$ & 74.97 & $\mathrm{Cu}(8.048)$ & $40^{*}$ & 5 & 6.4 \\
\hline $\mathrm{Fe}$ & 6.40 & $\mathrm{Cr}(5.414)$ & $8^{* *}$ & $0.7 * *$ & 4.8 \\
\hline
\end{tabular}

* poor signal-to-noise ratio; unrealiable peak detection

**exposure time required to obtain the deviation of 0.5

Further perspectives. As the size of the MYTHEN 1K detector reported to be main disadvantages, a development of a compact system, containing only 640 strips is in progress. Shorter silicon strips, enabling experiments on low energies (Ti-radiation) are also foreseen. As the detector can measure both low and high intensity peaks in the same scan $\left(1: 1.6 \times 10^{7}\right)$, further test of MYTHEN $1 \mathrm{~K}$ will include retained austenite measurements, where the limit is $<1 \%$ in aerospace applications. Due to short readout time resulting in high frame rate in combination with high quantum efficiency stress mapping of device under tests seems to be reasonable application as well as in situ process control.

\section{References}

[1] Koistinen, D.P. and R.E. Marburger, Trans. ASM, 51, 537 (1959).

[2] Ogilvey, R.E., M.S. Thesis, MIT, 1952.

[3] M.E. Fitzpatrick, A. Fry, P. Holdway, F.A. Kandil, J. Shackleton, L. Suominen, Determination of residual stresses by X-ray diffraction-Issue 2, Measurement good Practice No. 52 (2005)

[4] R. Jenkins, R.L. Snyder, Introduction to X-ray powder diffractometry, John Wiley\&Sons, 1996.

[5] Ch. Brönnimann, R. Baur, E.F. Eikenberry, S. Kohut, M. Lindner, B. Schmitt, R. Horisberger, A pixel read-out chip for the PILATUS project, Nucl. Instr. And Meth. A465 (2001) 235

[6] Ch. Brönnimann, E.F. Eikenberry, B. Henrich, R. Horisberger, G. Huelsen, E. Pohl, B. Schmitt, C. Schulze-Briese, M. Suzuki, T. Tomizaki, H. Toyokawa and A. Wagner, The PILATUS 1M detector, J. Synchrotron Rad. (2006). 13, 120-130

[7] B. Schmitt, Ch. Brönnimann, E.F. Eikenberry, F. Gozzo, C. Hörmann, R. Horisberger, B. Patterson, Mythen detector system, Nucl. Inst. Meth. Phy. Res A501 (2003), 267-272

[8] A. Bergamaschi, A. Cervellino, R. Dinapoli, F. Gozzo, B. Henrich, I. Johnson, P. Kraft, A. Mozzanica, B. Schmitt, X. Shi, The MYTHEN detector for X-ray powder diffraction experiments at the Swiss Light Source, J. Synch. Rad. 17 (2010), 653-668

[9] Z. Budrovic, H. Van Swygenhoven, P.M. Derlet, S. Van Petegem, B. Schmitt, Plastic Deformation with reversible peak broadening in nanocrystalline nickel, Science 304 (2004) 273

[10] V. Kostov, J. Gibmeier, F. Wilde, P. Staron, R. Rössler, A. Wanner, Fast in situ phase and stress analysis during laser surface treatment: A synchrotron x-ray diffraction approach, Rev. Sci. Instrum. 83 (2012) 115101

[11] J. Altenkirch, J. Gibmeier, V. Kostov, Th. Kannengiesser, S. Doyle, A. Wanner, Time- and temperature-resolved synchrotron $\mathrm{X}$ ray diffraction, The journal of Strain Analysis for engineering Design 46 (2011), 563-579 